\title{
A Procedure to Identify the Modal and Physical Parameters of a Classically Damped System under Seismic Motions
}

\author{
M. De Angelis ${ }^{1}$ and M. Imbimbo ${ }^{2}$ \\ ${ }^{1}$ Department of Structural and Geotechnical Engineering, Sapienza University of Roma, 00184 Rome, Italy \\ ${ }^{2}$ Department of Mechanics, Structures, and Environment, University of Cassino, 03043 Cassino, Italy \\ Correspondence should be addressed to M. Imbimbo, mimbimbo@unicas.it
}

Received 7 June 2011; Accepted 6 September 2011

Academic Editor: Luc Gaudiller

Copyright (C) 2012 M. De Angelis and M. Imbimbo. This is an open access article distributed under the Creative Commons Attribution License, which permits unrestricted use, distribution, and reproduction in any medium, provided the original work is properly cited.

\begin{abstract}
The purpose of this paper is to discuss a methodology for determining some modal parameters (frequencies, damping ratios, and seismic eigenvectors) and, under certain hypotheses, the physical matrices of a general structure with proportional damping and subjected to seismic loads. The procedure is based on a time-domain state space formulation from which the modal parameters, including the seismic eigenvectors, and the complex eigenvectors of the system can be derived also in the case of a limited set of instrumentations. The conditions to normalize the eigenvectors are then illustrated and, finally, applied to derive the secondorder matrices of the system. The proposed procedure is applied to some numerical examples also in the case of noise-polluted measurements and to an experimental investigation performed on a four-story steel frame subjected to earthquake excitations. An optimization procedure to improve the prediction of the first-order modal parameters of the system is also discussed and experimentally proved.
\end{abstract}

\section{Introduction}

System identification (SI) has shown a growing development in civil engineering during the last decades. A central issue of SI, especially within the field of civil engineering constructions, concerns the set of input/output experimental data with a particular emphasis on two main aspects: the number and the types of measurements (displacements, velocities, and accelerations) and the category of external actions to use in the process. Both the aspects are, in fact, related to the large-scale dimensions of civil engineering systems. The first aspect is widely discussed in $[1,2]$ where the minimum number of sensors and/or actuators required for a complete identification of a system is defined, in the case of input forces. Concerning the second aspect, Brownjohn [3] provides a clear discussion of the problem with specific reference to the external actions used in the dynamic tests on civil structures. In particular, these actions can be artificial forces applied to the structural system, such as those induced by a shaker or a vibrodine, and ambient vibrations, such as those due to traffic, earthquakes, and wind.
The use of forced vibration test data allows to identify the system's modal parameters and, also, retrieve the normalized eigenvectors, the mass, stiffness, and damping matrices of the system. The main drawback of the forced vibration test is that it requires large machineries which are often very expensive and cumbersome especially for large-scale structures such as civil constructions. A way to overcome this shortcoming is to adopt ambient vibration tests which use ambient actions always acting on the structures. The use of ambient vibration test data allows to identify the system's modal parameters as well. A comprehensive state-of-the-art review on fundamentals of system identification in structural dynamics in the case of environmental loads is reported in Imai et al. [4]. One common type of ambient vibrations in civil constructions is the earthquake excitations. In this field, some contributions, although the list is not exhaustive, are Ghanem and Shinozouka [5] and Shinozouka and Ghanem [6], which review and apply some structural identification algorithms for systems subjected to earthquake excitations, Beck and Jennings [7] and Mc Verry [8], which present an output-error method, respectively, in the time and frequency domain, and [9-11] 
which concern the identification of frequencies and dampings. In the case of the assumption of shear type buildings, some interesting contributions are the work by Yuan et al. [12] which provides a procedure to retrieve the normalized eigenvectors and the mass, stiffness, and damping matrices of the system, and Takewaki and Nakamura [13] which proposes a method of stiffness-damping simultaneous identification of the system using limited earthquake records. One of the more recent approaches in SI is based on a timedomain first-order formulation which provides the complex modal parameters of the system and a state space model capable of reproducing the input/output mapping of the system. These approaches are mostly used in control theory but notable contributions are also found in the field of identification $[1,2,14,15]$. Some of these studies specifically concerning systems subjected to earthquake loadings are Luş et al. [16] which identifies the modal parameters of the analyzed systems including the modal shapes and Fraraccio et al. [17] which presents an experimental study of different algorithms for the health monitoring of a shear-type system.

This paper discusses a methodology for determining some modal parameters (frequencies, damping ratios, and seismic eigenvectors) and, under certain hypotheses, the physical matrices of a general structure (not a shear-type one as usually analyzed in available literature) with proportional damping and subjected to seismic loads. In particular, the methodology is based on a time-domain first-order state representation using the well-known algorithm ERA/OKID (Eigensystem Realization Algorithm/Observer Kalman filter Identification) on the basis of the procedure discussed in [16, 18]. It allows, first, to identify the complex eigenvectors and the modal parameters of the system including, beyond the frequencies and the damping ratios, the seismic eigenvectors $[19,20]$, sometimes also named effective participation factors $[7,8]$. Then, the procedure is able to provide the conditions to normalizing the eigenvectors and, hence, deriving the second-order matrices of the system as reported in De Angelis et al. [1]. The significance of the proposed methodology consists, first, in the fact that the seismic eigenvectors are independent of the normalization of the mode shapes, and, hence, they can be determined whatever the number of sensors is and with no information and restrictions about the physical model of the system. This means that the seismic eigenvectors can represent a useful tool for health monitoring and of significance in updating models where they provide, jointly with the frequencies and dampings, additional information for validating the model. The other point of interest of the proposed procedure is that, in the case of full set of instrumentations, it provides the conditions to normalizing the eigenvectors and, hence, deriving the second-order matrices of the system without any restrictions about the system behaviour (such as the shear-type assumption). The methodology is discussed theoretically and, then, applied to some numerical examples which have shown the efficacy of the procedure also in the case of noise-polluted measurements. Finally, the procedure is also applied to an experimental test performed on a four-story steel frame subjected to earthquake excitations.

\section{Some Background}

Let us consider a seismically loaded $N$ degree of freedom structural system whose second-order equations of motion can be written as follows:

$$
\begin{gathered}
\mathbf{M} \ddot{\mathbf{x}}(t)+\mathbf{L} \dot{\mathbf{x}}(t)+\mathbf{K x}(t)=-\mathbf{M U} \ddot{x}_{g}(t), \\
\mathbf{y}(t)=\mathbf{c}_{p} \mathbf{x}(t),
\end{gathered}
$$

where $\mathbf{x}(t)$ is the $N \times 1$ vector of generalized nodal displacements relative to the ground and the notation $(\cdot)$ indicates the differentiation with respect to time; $\mathbf{M}, \mathbf{L}$, and $\mathbf{K}$ are the $N \times N$ mass, damping and stiffness matrices of the system, $\mathrm{U}$ is the $N \times 1$ influence vector, with all terms equal to $\mathbf{1}$, and $\ddot{x}_{g}(t)$ is the ground acceleration. The $m \times 1$ output vector $\mathbf{y}(t)$ contains the $m$ time histories of the displacement output measurements and $\mathbf{c}_{p}$ is the matrix which selects the degrees of freedom where the displacements are known. In the case of accelerations output measurements, equations similar to the second of (1) can be written with a matrix $\mathbf{c}_{a}$ used in place of the matrix $c_{p}$ to select the degrees of freedom where the accelerations are known.

By using the modal transformation $\mathbf{x}(t)=\phi \mathbf{q}(t)$, being $\phi=\left[\phi_{1} \phi_{2} \ldots \phi_{N}\right]$ the eigenvector matrix and $\mathbf{q}(t)$ the modal coordinates, (1), in modal coordinates, result in the following:

$$
\begin{gathered}
\ddot{\mathbf{q}}(t)+\boldsymbol{\varepsilon} \dot{\mathbf{q}}(t)+\mathbf{\Omega q}(t)=-\phi^{T} \mathbf{M U} \ddot{x}_{g}(t), \\
\mathbf{y}(t)=\mathbf{c}_{p} \phi \mathbf{q}(t),
\end{gathered}
$$

with $\phi^{T} \mathbf{M} \phi=\mathbf{I}, \boldsymbol{\phi}^{T} \mathbf{K} \boldsymbol{\phi}=\mathbf{\Omega}=\operatorname{diag}\left(\omega_{k}^{2}\right)$.

If the system is classically damped, the matrix $\varepsilon$ is diagonal, that is, $\boldsymbol{\varepsilon}=\operatorname{diag}\left(\varepsilon_{k}\right)$ and $\varepsilon_{k}=\boldsymbol{\phi}_{k}^{T} \mathbf{L} \boldsymbol{\phi}_{k}=2 \zeta_{k} \omega_{k}$ with $\zeta_{k}$ the $k$ th damping ratio. In this case, the Laplace transform of (2) provides the following, for the $j$ th dof:

$$
y_{j}(s)=-\sum_{k=1}^{N} \frac{\phi_{j k} P_{k}}{s^{2}+\varepsilon_{k} s+\Omega_{k}} \ddot{x}_{g}(s)=-\sum_{k=1}^{N} \frac{\theta_{j k}}{s^{2}+\varepsilon_{k} s+\Omega_{k}} \ddot{x}_{g}(s),
$$

where $P_{k}=\phi_{k}^{T} \mathbf{M U} / \phi_{k}^{T} \mathbf{M} \phi_{k}$ is the $k$ th modal participation factor and $\theta_{j k}=\phi_{j k} P_{k}$ is the effective modal participation factor at position $j$ of mode $k[7,8]$ or also named the seismic eigenvector $\boldsymbol{\theta}_{k}[19,20]$. The components $\theta_{j k}$ satisfy the following relationship:

$$
\theta \mathbf{U}=\mathbf{U} .
$$

By introducing the state vector $\boldsymbol{\eta}(t)=\left\{\mathbf{x}(t)^{T} \dot{\mathbf{x}}(t)^{T}\right\}^{T},(1)$ can be transformed to the symmetrical first-order form:

$$
\begin{gathered}
\mathbf{A} \dot{\boldsymbol{\eta}}(t)+\mathbf{B} \boldsymbol{\eta}(t)=\mathbf{E} \ddot{x}_{g}(t), \\
\mathbf{y}(t)=\mathbf{C} \boldsymbol{\eta}(t)+\mathbf{D} \ddot{x}_{g}(t),
\end{gathered}
$$

where the matrices $\mathbf{A}, \mathbf{B}, \mathbf{E}, \mathbf{C}$, and $\mathbf{D}$ are given by:

$$
\begin{gathered}
\mathbf{A}=\left[\begin{array}{cc}
\mathbf{L} & \mathbf{M} \\
\mathbf{M} & \mathbf{O}
\end{array}\right] ; \quad \mathbf{B}=\left[\begin{array}{cc}
\mathbf{K} & \mathbf{O} \\
\mathbf{O} & -\mathbf{M}
\end{array}\right] ; \quad \mathbf{E}=\left[\begin{array}{c}
-\mathbf{M U} \\
\mathbf{O}
\end{array}\right] ; \\
\mathbf{C}=\left[\begin{array}{cc}
\mathbf{c}_{p} & \mathbf{O}
\end{array}\right] ; \quad \mathbf{D}=[\mathbf{O}],
\end{gathered}
$$

being $\mathbf{O}$ the zero vector or matrix. 
The eigenvalue problem associated to (5) provides the complex eigenvalues $\lambda_{i}$ and eigenvectors $\psi_{i}(i=1,2, \ldots, 2 N)$ which can be arranged respectively, in the square matrix $\Lambda=$ $\operatorname{diag}\left(\lambda_{1} \lambda_{2} \cdots \lambda_{2 N}\right)$, of dimension $2 N \times 2 N$, and in the eigenvector matrix $\psi=\left[\psi_{1} \psi_{2} \cdots \psi_{2 N}\right]$ of dimension $N \times 2 N$. It results in $\lambda_{k}^{*}=\lambda_{N+k}$ and $\psi_{k}^{*}=\psi_{N+k}$ (the superscript $\left(^{*}\right.$ ) indicates the complex conjugate) with $k=1,2, \ldots N$.

Considering the following matrix $\boldsymbol{\Psi}=\left[\boldsymbol{\psi}^{T}(\boldsymbol{\psi} \boldsymbol{\Lambda})^{T}\right]^{T}$ the complex eigenvector can be normalized according to the following relations:

$$
\boldsymbol{\Psi}^{T} \mathbf{A} \boldsymbol{\Psi}=\boldsymbol{v} ; \quad \boldsymbol{\Psi}^{T} \mathbf{B} \boldsymbol{\Psi}=-\boldsymbol{\nu} \boldsymbol{\Lambda},
$$

where the matrix $\boldsymbol{v}$ can be defined alternatively as follows:

$\boldsymbol{\nu}=\boldsymbol{\Lambda}-\boldsymbol{\Lambda}^{*}$; in this case the matrix $\boldsymbol{\nu}$ can be related to the natural modes $\phi$ previously defined and, in particular, equal to them when the system is classically damped.

$\boldsymbol{v}=\mathbf{I}$; in this case, when the damping is proportional, the eigenvalues have the same real and imaginary parts.

In this paper it is assumed $\boldsymbol{\nu}=\mathbf{I}$.

By using the modal transformation $\boldsymbol{\eta}(t)=\boldsymbol{\Psi} \boldsymbol{\xi}(t)$, with $\xi(t)$ expressing the modal coordinates, (5), in modal coordinates, result in the following:

$$
\begin{gathered}
\dot{\boldsymbol{\xi}}(t)=\boldsymbol{\Lambda} \boldsymbol{\xi}(t)-\boldsymbol{\Psi}^{T} \mathbf{M U} \ddot{x}_{g}(t)=\Lambda \boldsymbol{\xi}(t)-\mathbf{Q} \ddot{x}_{g}(t), \\
\mathbf{y}(t)=\mathbf{c}_{p} \Psi \boldsymbol{\Psi}(t),
\end{gathered}
$$

where the vector $\mathbf{Q}$ is defined as $\mathbf{Q}=\boldsymbol{\Psi}^{T} \mathbf{M U}$, being $Q_{k}=$ $\psi_{k}^{T} \mathbf{M U}=Q_{N+k}^{*}$.

The Laplace transform of (8) provides the following, for the $j$ th dof:

$$
\begin{aligned}
& y_{j}(s) \\
& =-\sum_{k=1}^{N}\left(\frac{\left(\psi_{j k} Q_{k}+\psi_{j k}^{*} Q_{k}^{*}\right) s+\left(\psi_{j k} \lambda_{k}^{*} Q_{k}+\psi_{j k}^{*} \lambda_{k} Q_{k}^{*}\right)}{s^{2}-\left(\lambda_{k}+\lambda_{k}^{*}\right) s+\lambda_{k} \lambda_{k}^{*}}\right) \ddot{x}_{g}(s),
\end{aligned}
$$

which is valid for both classically and nonclassically damped systems.

The frequencies $\Omega_{k}$ and damping ratios $\zeta_{k}$ can be evaluated through the following expressions: $\Omega_{k}=\sqrt{\alpha_{k}^{2}+\beta_{k}^{2}}$, $\zeta_{k}=-\alpha_{k} / \Omega_{k}$ where the $k$ th eigenvalue, $\lambda_{k}$, and its conjugate, $\lambda_{k}^{*}=\lambda_{N+k}$, are given by $\lambda_{k, N+k}=\alpha_{k} \pm i \beta_{k}(i=\sqrt{-1}$ is the imaginary number).

It is worthy to mention that, if the eigenvectors are normalized through (7), then the mass, stiffness, and damping matrices can be expressed as functions of the complex eigenvalues and eigenvectors, De Angelis et al. [1], as follows:

$$
\begin{gathered}
\mathbf{M}=\left(\boldsymbol{\psi} \boldsymbol{\Lambda} \boldsymbol{\psi}^{T}\right)^{-1} ; \quad \mathbf{K}=-\left(\boldsymbol{\psi} \boldsymbol{\Lambda}^{-1} \boldsymbol{\psi}^{T}\right)^{-1} ; \\
\mathbf{L}=-\mathbf{M} \boldsymbol{\psi} \boldsymbol{\Lambda}^{2} \boldsymbol{\psi}^{T} \mathbf{M},
\end{gathered}
$$

with the eigenvectors satisfying the following conditions:

$$
\psi \psi^{T}=\mathbf{0}
$$

For general damping assumptions the relationship between the modal parameters of the second- and the firstorder models are quite difficult to derive, Chen et al. [11], while in the case of classical damping, the two representations are related through simple relationships. In fact, by comparing (3) and (9) it results in the following:

$$
\begin{gathered}
0=\psi_{j k} Q_{k}+\psi_{j k}^{*} Q_{k}^{*}, \\
\theta_{j k}=\psi_{j k} \lambda_{k}^{*} Q_{k}+\psi_{j k}^{*} \lambda_{k} Q_{k}^{*}, \\
s^{2}+\varepsilon_{k} s+\Omega_{k}=s^{2}-\left(\lambda_{k}+\lambda_{k}^{*}\right) s+\lambda_{k} \lambda_{k}^{*} .
\end{gathered}
$$

The quantities $\epsilon_{j k}$ and $\epsilon_{j k}^{*}$, given by the second expression of (12), can be rewritten as follows:

$$
\boldsymbol{\epsilon}=\boldsymbol{\psi} \boldsymbol{\Lambda}^{*} \operatorname{diag}\left(Q_{k}, Q_{k}^{*}\right) .
$$

Similarly to the matrix $\boldsymbol{\theta}$, also the matrix $\boldsymbol{\epsilon}$ is independent on the transformation and, thus, satisfies a relationship analogous to (4), that is,

$$
\epsilon \mathrm{U}=\mathrm{U}
$$

which is valid for general damping assumptions.

\section{Identification of Modal and Physical Parameters}

This section is devoted to discuss a methodology for identifying the natural frequencies, the modal damping ratios, the seismic eigenvectors, and, in some cases, the physical matrices of a linear classically damped structure subjected to seismic ground motion. The methodology is based on a time-domain first-order state representation and, in detail, identifies the following quantities: (i) first-order model; (ii) frequencies, dampings, and seismic eigenvectors; (iii) physical parameters (stiffness, damping, and mass matrices).

(i) Identification of the First-Order Model. The first phase of this methodology consists of identifying the first-order state space model of the system from general input/output data using an Eigensystem Realization Algorithm/Observer Kalman filter Identification- (ERA/OKID-) based approach $[16,21]$. The identified modal model is expressed as follows (De Angelis et al. [1]):

$$
\begin{gathered}
\dot{\xi}_{1}(t)=\Lambda \xi_{1}(t)+\mathbf{B}_{1} \ddot{x}_{g}(t), \\
\mathbf{y}(t)=\mathbf{C}_{1 p} \xi_{1}(t),
\end{gathered}
$$

where $\Lambda, \mathbf{B}_{1}$ and $\mathbf{C}_{1 p}$ are complex matrices given by

$$
\begin{gathered}
\Lambda=\mathbf{T}_{0}^{-1} \mathbf{A}_{0} \mathbf{T}_{0}, \\
\mathbf{B}_{1}=\mathbf{T}_{0}^{-1} \mathbf{B}_{0}, \\
\mathbf{C}_{1 p}=\mathbf{C}_{0 p} \mathbf{T}_{0} .
\end{gathered}
$$

In (16), $\mathbf{A}_{0}, \mathbf{B}_{0}$, and $\mathbf{C}_{0 p}$ are the identified continuous state matrices, and $\mathbf{T}_{0}$ is a transformation matrix which relates the two representations of the first- and second-order of the model, De Angelis et al. [1]. The matrix $\Lambda$ contains the complex eigenvalues, $\lambda_{k}$ and $\lambda_{k}^{*}$, and the matrix $\mathbf{C}_{1 p}$ contains the complex eigenvectors of the system. 
It is worthy to remember that the relationship between the identified matrix related to the absolute accelerations, $\mathbf{C}_{1 a}$, and that related to the displacements, $\mathbf{C}_{1 p}$, is given by

$$
\mathrm{C}_{1 p}=\mathrm{C}_{1 a} \boldsymbol{\Lambda}^{-2}
$$

(ii) Identification of Frequencies, Dampings, and Seismic Eigenvectors. Once the complex eigenvalues $\lambda_{k}$ and $\lambda_{k}^{*}$ are identified, the frequencies and dampings parameters of the system can be derived from (12), that is,

$$
\Omega_{k}=\lambda_{k} \lambda_{k}^{*}=\omega_{k}^{2} ; \quad-\left(\lambda_{k}+\lambda_{k}^{*}\right)=2 \zeta_{k} \omega_{k} .
$$

Once the matrices $\mathbf{C}_{1 p}$ and $\mathbf{B}_{1}$ are identified through (15), the seismic eigenvectors can be derived from (12) that is,

$$
\theta_{j k}=C_{1 p j k} \lambda_{k}^{*} B_{1 k}+C_{1 p j k}^{*} \lambda_{k} B_{1 k}^{*} .
$$

It is worthy to remind that the seismic eigenvectors identified at this stage has a double advantage. First they are independent of the normalization of the normal modes and, thus, can be determined also in case of limited set of instrumentations and without any information about the physical model of the system. In addition, they constitute a set of modal parameters which are of primary interest in many system identification applications such as finite element models or health monitoring problems. In fact, in the first case, they provide further information for the model validation which can be added to frequencies and damping; in health monitoring, they can be efficiently used in detecting damage and its location.

(iii) Identification of the Physical Parameters. The physical parameters of the system can be obtained by (10) if the complex eigenvectors contained in the matrix $\mathbf{C}_{1 p}$ are normalized through (7). To this purpose the conditions, in terms of number of sensors and information about the mass of the system, to normalizing the eigenvectors and deriving the second-order matrices are discussed.

Let us assume that the complex eigenvectors contained in the matrix $\mathbf{C}_{1 p}$ and the normalized complex eigenvector $\psi$ are related through a scaling matrix $\boldsymbol{\tau}$ such that,

$$
\psi=\mathbf{C}_{1 p} \tau,
$$

where $\tau$ is a complex diagonal matrix with terms such that $\tau_{k}=\tau_{N+k}^{*}$ which contains the unknown scaling factors.

Using the scaling condition of (20), written as follows:

$$
\mathbf{M}^{-1}=\psi \Lambda \psi^{T}
$$

and in the common assumption of a diagonal mass matrix, (21) provides $n e_{1}=m(m+1) / 2-m$ equations expressing the zero off-diagonal terms. Equation (11) provides $n e_{2}=m(m+$ $1) / 2$ equations, where $m$ is the number of measurement points. The total number of equations is $n e_{T}$ given by $n e_{T}=$ $m^{2}$. Then, the eigenvectors can be normalized only when

$$
n e_{T}+n_{m} \geq 2 N
$$

where $n_{m}(\geq 1)$ is the number of known masses connected to the measured dofs. If the condition is satisfied, it is possible to determine the following quantities:
(1) the transformation matrix $\tau$, (2) the normalized eigenvectors $\psi$, and (3) the physical matrices $\mathbf{M}, \mathbf{L}$, and $\mathbf{K}$.

Using (20), (11) and (21) become:

$$
\begin{gathered}
\sum_{k=1}^{N}\left(c_{i k} c_{j k} \tau_{k}^{2}+c_{i k}^{*} c_{j k}^{*} \tau_{k}^{* 2}\right)=0, \\
\sum_{k=1}^{N}\left(c_{i k} c_{j k} \lambda_{k} \tau_{k}^{2}+c_{i k}^{*} c_{j k}^{*} \lambda_{k}^{*} \tau_{k}^{* 2}\right)=m_{i j}^{-1},
\end{gathered}
$$

with $i, j=1,2, \ldots, m$. When $i=j$, the second set of (23) is utilized only if the mass $m_{i i}$ is known. The system of equations (23) can be rewritten in the following form:

$$
\mathbf{Z v}=\mathbf{t}
$$

where $\mathbf{v}$ is the vector containing the unknown terms $\tau_{k}^{2}$ and $\tau_{k}^{* 2}\left(v_{k}=\tau_{k}^{2}\right.$ and $\left.v_{k}^{*}=\tau_{k}^{* 2}\right), \mathrm{Z}$ is the coefficients matrix, $\mathbf{t}$ is a vector that contains all the known quantities. If all masses are unknown, $\mathbf{t}$ is the zero vector and the system is homogeneous. Then the solution depends on a single parameter which can be determined only if one mass of the measured dofs is known. Generally the system (24) is characterized by a number of equations greater than the number of unknowns and the vector $\mathbf{v}$ is given by $\mathbf{v}=\mathbf{Z}^{+} \mathbf{t}$, where $\mathbf{Z}^{+}$is the pseudoinverse of the matrix $\mathbf{Z}$. Once the vector $\mathbf{v}$ is known, the matrix $\boldsymbol{\tau}$ can be derived.

In the case of a complete set of measurements, that is $m=$ $N$, the physical matrices of the system can be derived by (10) with $\psi=\mathrm{C}_{1 p} \tau$.

In the case of incomplete measurements, that is, $m<$ $N$, it is still possible to normalize the eigenvectors if the condition (22) is satisfied. However, only reduced forms of the physical matrices are possible to be derived, Luş et al. [2]. The approximation of these reduced forms depends on the damping system assumptions.

In the case of classically damped systems, the modes of vibration $\phi$ of the system can be derived from the complex eigenvectors $\psi$. Then, the $m \times m$ "reduced" form of the mass, damping, and stiffness matrices are as follows:

$$
\begin{gathered}
\widehat{\mathbf{M}}=\left(\hat{\boldsymbol{\phi}}^{+}\right)^{T}\left(\hat{\boldsymbol{\phi}}^{+}\right) ; \quad \hat{\mathbf{L}}=\left(\hat{\boldsymbol{\phi}}^{+}\right)^{T} \boldsymbol{\varepsilon}\left(\hat{\boldsymbol{\phi}}^{+}\right) ; \\
\widehat{\mathbf{K}}=\left(\hat{\boldsymbol{\phi}}^{+}\right)^{T} \boldsymbol{\Omega}\left(\hat{\boldsymbol{\phi}}^{+}\right),
\end{gathered}
$$

where $\hat{\phi}$ is the $m \times N$ matrix containing the identified eigenvectors and $\hat{\phi}^{+}$is its pseudoinverse. The matrices (25) are the partitions of the "full-order" physical matrices referred to the measured degrees of freedom.

In the case of nonclassically damped systems, the reduced forms of the physical matrices are expressed in terms of the complex eigenvectors $\hat{\psi}$, of dimension $m \times 2 N$, as follows:

$$
\begin{gathered}
\widehat{\mathbf{M}}=\left(\hat{\boldsymbol{\psi}} \boldsymbol{\Lambda} \hat{\psi}^{T}\right)^{-1}, \quad \hat{\mathbf{L}}=-\widehat{\mathbf{M}} \hat{\boldsymbol{\psi}} \boldsymbol{\Lambda}^{2} \widehat{\boldsymbol{\psi}}^{T} \widehat{\mathbf{M}} \\
\hat{\mathbf{K}} \simeq\left(\widehat{\mathbf{L}} \widehat{\mathbf{M}}^{-1} \hat{\mathbf{L}}-\widehat{\mathbf{M}} \hat{\boldsymbol{\psi}} \boldsymbol{\Lambda}^{3} \widehat{\boldsymbol{\psi}}^{T} \widehat{\mathbf{M}}\right) .
\end{gathered}
$$

The "reduced" forms given by the first two equations (26) are exact and provide the known partitions of the "fullorder" mass and damping matrices of the system. On the 
contrary the "reduced" form given by the third equation (26) provides only an approximation of the exact partition of the "full-order" stiffness matrix, Luş et al. [2].

3.1. The Case of All Unknown Masses. The assumption of having at least one known mass at the measured degrees of freedom to identify the physical properties of the system can be easily verified if the procedure is applied to buildings where the evaluation of the floor mass is quite simple.

However, the hypothesis of a known mass can be also removed in the case of damage detection problems and health monitoring models. In fact, if all the masses are unknown, it is possible to determine a transformation matrix $\tau^{\prime}$ such that

$$
\tau_{k}^{2}=m_{l}^{-1} \tau_{k}^{\prime 2}
$$

where $m_{l}$ is the unknown mass related to the $l$ th dof. Then (23) becomes

$$
\begin{gathered}
\sum_{k=1}^{N}\left(c_{i k} c_{j k} \tau_{k}^{\prime 2}+c_{i k}^{*} c_{j k}^{*} \tau_{k}^{\prime * 2}\right)=0, \\
\sum_{k=1}^{N}\left(c_{i k} c_{j k} \lambda_{k} \tau_{k}^{\prime 2}+c_{i k}^{*} c_{j k}^{*} \lambda_{k}^{*} \tau_{k}^{\prime * 2}\right)=\delta_{i j},
\end{gathered}
$$

with the condition that the second set of equations (28) includes only the term with $i=j=l$ in addition to the terms with $i \neq j$. The solution of the system (28) provides the unknown matrix $\boldsymbol{\tau}^{\prime}$.

Then, using (20) and (27), the eigenvectors are given by

$$
\psi=m_{l}^{-0.5} \mathbf{C}_{1 p} \boldsymbol{\tau}^{\prime}=m_{l}^{-0.5} \psi^{\prime},
$$

and the "full-order" physical matrices $(m=N)$ are expressed through the following expressions:

$$
\begin{gathered}
\mathbf{M}=m_{l}\left(\mathbf{C}_{1 p} \boldsymbol{\tau}^{\prime} \boldsymbol{\Lambda} \boldsymbol{\tau}^{\prime T} \mathbf{C}_{1 p}^{T}\right)=m_{l} \mathbf{M}^{\prime}, \\
\mathbf{K}=-m_{l}\left(\mathbf{C}_{1 p} \boldsymbol{\tau}^{\prime} \boldsymbol{\Lambda}^{-1} \boldsymbol{\tau}^{\prime T} \mathbf{C}_{1 p}^{T}\right)=m_{l} \mathbf{K}^{\prime}, \\
\mathbf{L}=-m_{l}\left(\mathbf{M}^{\prime} \mathbf{C}_{1 p} \boldsymbol{\tau}^{\prime} \boldsymbol{\Lambda}^{2} \boldsymbol{\tau}^{\prime T} \mathbf{C}_{1 p}^{T}\right) \mathbf{M}^{\prime}=m_{l} \mathbf{L}^{\prime} .
\end{gathered}
$$

In the case of incomplete measurements, that is, $m<N$, it is still possible to derive expressions analogous to (25) and (26) and containing the unknown mass $m_{l}$.

It is interesting to observe that the identified matrices (30) depend linearly on the unknown mass $m_{l}$. This could be useful in the case of finite element updating models as well as in damage detection processes. In this last case the comparison between the system's state before and after damage would be independent on the unknown mass $m_{l}$ if it is assumed constant.

\section{Optimization of Modal Parameters}

The identification process is generally affected by errors which depend either on modelling errors or noise pollution. The prediction of the modal parameters can be improved by minimizing the following objective function:

$$
f(\mathbf{p}, t)=[\mathbf{y}(t)-\mathbf{y}(\mathbf{p}, t)]^{T}[\mathbf{y}(t)-\mathbf{y}(\mathbf{p}, t)],
$$

where $\mathbf{y}(t)$ is the measured data, and $\mathbf{y}(\mathbf{p}, t)$ is the predicted data, being $\mathbf{p}$ a vector containing the modal parameters to be optimized. The parameters $p_{i}$ already identified and referred to the eigenvectors, can be constrained through (11) and (14) and the condition of diagonal mass (21); if the system is classically damped the parameters $p_{i}$ can also be constrained through (12).

The predicted data $\mathbf{y}(\mathbf{p}, t)$ included in (31) can be obtained by considering the identified first-order model:

$$
\begin{gathered}
\dot{\boldsymbol{\xi}}(t)=\boldsymbol{\Lambda} \boldsymbol{\xi}(t)+\mathbf{Q} \ddot{x}_{g}(t), \\
\mathbf{y}(t)=\hat{\psi} \boldsymbol{\Lambda}^{2} \boldsymbol{\xi}(t) .
\end{gathered}
$$

The McMillan transformation, Luş [18], applied to (32) provides a first-order model with real matrices and vectors given by:

$$
\begin{gathered}
\dot{\boldsymbol{\xi}}(t)=\mathbf{A}_{M c} \boldsymbol{\xi}(t)+\mathbf{B}_{M c} \ddot{x}_{g}(t), \\
\mathbf{y}(t)=\mathbf{C}_{M c} \boldsymbol{\xi}(t),
\end{gathered}
$$

where the matrix $\mathbf{A}_{M c}$ has the following expression:

$$
\mathbf{A}_{M c}=\left[\begin{array}{cc}
\mathbf{O} & \mathbf{I} \\
-\operatorname{diag}\left(\Omega_{k}\right) & \operatorname{diag}\left(\varepsilon_{k}\right)
\end{array}\right],
$$

with $\mathbf{O}$ and $\mathbf{I}$ the zero and unity $N \times N$ matrices, $\mathbf{B}_{M c}$ an $N \times 1$ vector and $\mathbf{C}_{M c}$ an $m \times N$ matrix.

To perform the optimization, the system (33) is transformed from the continuous to the discrete time:

$$
\begin{gathered}
\mathbf{x}(k+1)=\boldsymbol{\Phi}_{M c} \mathbf{x}(k)+\boldsymbol{\Gamma}_{M c} \ddot{x}_{g}(k), \\
\mathbf{y}(k)=\mathbf{C}_{M c} \mathbf{x}(k),
\end{gathered}
$$

where $k$ is a time index, $\boldsymbol{\Phi}_{M c}$ is the discrete state transition matrix, $\boldsymbol{\Gamma}_{M c}$ is the discrete time input vector. It is worthy to underline that the system (35) in the discrete time is equivalent to the system (33) in the continuous time and it is used in the computational procedure. Using (35), (31) can be rewritten as follows:

$$
f(\mathbf{p}, k)=[\mathbf{y}(k)-\mathbf{y}(\mathbf{p}, k)]^{T}[\mathbf{y}(k)-\mathbf{y}(\mathbf{p}, k)] .
$$

\section{Applications}

5.1. Numerical Applications. The identification procedure is applied, De Angelis et al. [22], to the five degrees of freedom system illustrated in Figure 1. The data used in the identification are provided by numerical analyses.

The response of the system is expressed in terms of absolute accelerations at each degree of freedom and is evaluated through the finite element model subjected to the north-south component of the El Centro Earthquake $(18 / 5 / 1940)$. It is assumed $d t=0.02 \mathrm{sec}$ and a number of points equal to 1560 . The time history accelerations are, then, polluted with Gaussian, zero-mean, white noise sequences whose root mean square is equal to $10 \%$ of the unpolluted data. 
TABLE 1: Eigenvalues $\left(\times 10^{2}\right)$.

\begin{tabular}{lcccc}
\hline$\lambda_{1,6}$ & $\lambda_{2,7}$ & $\lambda_{3,8}$ & $\lambda_{4,9}$ & $\lambda_{5,10}$ \\
\hline$-0.0016 \mp 0.0781 i$ & $-0.0050 \mp 0.2506 i$ & $-0.0094 \mp 0.4681 i$ & $-0.0148 \mp 0.7381 i$ & $-0.0206 \mp 1.0298 i$ \\
\hline
\end{tabular}

TABLE 2: Matrix $\mathbf{B}_{1}\left(\times 10^{-2}\right)$.

\begin{tabular}{lcccc}
\hline$b_{1,6}$ & $b_{2,7}$ & $b_{3,8}$ & $b_{4,9}$ & $b_{5,10}$ \\
\hline$+0.7517 \pm 0.3543 i$ & $+0.3940 \pm 0.9553 i$ & $+0.9173 \pm 0.9067 i$ & $+1.1515 \pm 1.0904 i$ & $+1.5067 \pm 1.0867 i$ \\
\hline
\end{tabular}

Table 3: Matrix $\mathbf{C}_{1 p}\left(\times 10^{-3}\right)$.

\begin{tabular}{lcccc}
\hline $\mathbf{c}_{1,6}$ & $\mathbf{c}_{2,7}$ & $\mathbf{c}_{3,8}$ & $\mathbf{c}_{4,9}$ & $\mathbf{c}_{5,10}$ \\
\hline$+0.0677 \mp 0.1451 i$ & $-0.0445 \pm 0.0190 i$ & $+0.0163 \pm 0.0157 i$ & $+0.0056 \mp 0.0064 i$ & $+0.0010 \mp 0.0015 i$ \\
$+0.1848 \mp 0.3960 i$ & $-0.0829 \pm 0.0354 i$ & $+0.0112 \pm 0.0108 i$ & $-0.0032 \pm 0.0036 i$ & $-0.0016 \pm 0.0024 i$ \\
$+0.2954 \mp 0.6330 i$ & $-0.0593 \pm 0.0253 i$ & $-0.0133 \mp 0.0128 i$ & $-0.0035 \pm 0.0040 i$ & $+0.0015 \mp 0.0023 i$ \\
$+0.3808 \mp 0.8161 i$ & $+0.0145 \mp 0.0062 i$ & $-0.0137 \mp 0.0132 i$ & $+0.0059 \mp 0.0067 i$ & $-0.0009 \pm 0.0015 i$ \\
$+0.4354 \mp 0.9330 i$ & $+0.0952 \mp 0.0407 i$ & $+0.0175 \pm 0.0169 i$ & $-0.0034 \pm 0.0039 i$ & $+0.0004 \mp 0.0006 i$ \\
\hline
\end{tabular}

TABLE 4: Identified frequencies and damping coefficients.

\begin{tabular}{lccccc}
\hline Modes & 1 & 2 & 3 & 4 & 5 \\
\hline$f(\mathrm{~Hz})$ & 1.2429 & 3.9873 & 7.4486 & 11.748 & 16.393 \\
$\zeta(\%)$ & 2.000 & 2.000 & 2.000 & 2.000 & 2.000 \\
\hline
\end{tabular}

5.1.1. Registrations at All Floors. In this first case the accelerations at each floor and the ground acceleration are measured.

The ERA/OKID algorithm, Luş et al. [16], provides the identified matrices $\mathbf{A}_{0}, \mathbf{B}_{0}$, and $\mathbf{C}_{0 a}$; next, the matrices $\Lambda, \mathbf{B}_{1}$, and $\mathbf{C}_{1 p}=\mathbf{C}_{1 a} \boldsymbol{\Lambda}^{-2}$ are derived through (16) and reported in Table 1, 2, and 3. Using the eigenvalues of the firstorder system and applying (18), it is possible to identify the frequencies and damping coefficients of the system (Table 4) which are coincident with the exact ones. The complex eigenvectors $\psi$ obtained by (20) provide, through (13), the vector $\boldsymbol{\epsilon}$. Finally, the seismic eigenvectors $\boldsymbol{\theta}_{k}$ are evaluated through (19) and the results are reported in Table 5. Figure 2 shows the first two identified seismic eigenvectors $\boldsymbol{\theta}_{k}$.

The exact values of the modal parameters have been obtained and conditions (4) and (14) are satisfied for $\theta_{j k}$ and $\epsilon_{j k}$.

Once the modal parameters are obtained, the physical matrices of the system can be obtained only if the complex eigenvectors are normalized. In this case, under the assumption of full measurements (i.e., $m=5$ ) and the knowledge of the mass at the first degree of freedom (i.e., $n_{m}=$ 1 ), the condition (22) is satisfied and provides 26 available equations. The solution of the system (24), then, provides the vector $\mathbf{v}^{\prime}$ reported in Table 6.

The normalized and nonscaled eigenvectors $\psi^{\prime}$ (Table 7) are derived from (29).

From $\psi^{\prime}$, using the value of the mass $m_{1}=50 t$, it is possible to evaluate the transformation matrix and, then, the eigenvector $\psi$.
The real and imaginary parts of the eigenvectors being equal, the system is classically damped.

The vectors $\phi_{k}$ (Table 8 ) are then evaluated from the eigenvectors $\psi$. In Figure 2 the first two identified eigenvectors $\phi_{k}$ are also reported (to note the proportionality between the two eigenvectors).

The mass and stiffness matrices are, finally, derived from (10) and are given by:

$\mathbf{M}=\operatorname{diag}[50.0000 ; 40.0000 ; 40.0000 ; 40.0000 ; 30.0000]$,

$$
\mathbf{K}=10^{5}\left[\begin{array}{rrrrr}
+2.3120 & -1.3790 & +0.4300 & -0.0860 & +0.0140 \\
-1.3790 & +1.9130 & -1.2820 & +0.3860 & -0.0550 \\
+0.4300 & -1.2820 & +1.8440 & -1.1550 & +0.2530 \\
-0.0860 & +0.3860 & -1.1550 & +1.4400 & -0.6050 \\
+0.0140 & -0.0550 & +0.2530 & -0.6050 & +0.3970
\end{array}\right] .
$$

It is clear, then, that the proposed identification procedure provides, in the case of noise-free measurements, the exact values of the modal and physical parameters.

5.1.2. Registrations at 1 st, $3 \mathrm{rd}, 4$ th, and 5th Floors. The identification of the first-order model and modal parameters is not affected by the number of measurements and, thus, the frequencies and the damping coefficients are exactly the same obtained in the previous case (Table 4). Also the eigenvector $\boldsymbol{\theta}_{k}$ is the same except for the component related to the 2 nd dof where the data are missed.

In this case, of incomplete measurements $(m=4)$ and still one known mass at the first degree of freedom $\left(n_{m}=1\right)$, the condition (22) is still satisfied and the eigenvectors can still be normalized. The eigenvectors $\phi_{k}$ are, then, evaluated from the complex eigenvectors and are the same as the previous case except for the component related to the 2 nd 
TABle 5: Identified vectors $\boldsymbol{\theta}_{i}=\epsilon_{i}+\epsilon_{i}^{*}$.

\begin{tabular}{lccccc}
\hline $\boldsymbol{\theta}_{1}$ & $\boldsymbol{\theta}_{2}$ & $\boldsymbol{\theta}_{3}$ & $\boldsymbol{\theta}_{4}$ & $\boldsymbol{\theta}_{5}$ & $\sum_{k=1}^{5} \boldsymbol{\theta}_{j k}$ \\
\hline+0.2079 & +0.2507 & +0.2730 & +0.2002 & +0.0679 & 1.000 \\
+0.5674 & +0.4670 & +0.1885 & -0.1128 & -0.1100 & 1.000 \\
+0.9070 & +0.3341 & -0.2231 & -0.1237 & +0.1057 & 1.000 \\
+1.1693 & -0.0818 & -0.2303 & +0.2093 & -0.0665 & 1.000 \\
+1.3368 & -0.5361 & +0.2930 & -0.1203 & +0.0266 & 1.000 \\
\hline
\end{tabular}

TABLE 6: Vector $\mathbf{v}^{\prime T}$.

\begin{tabular}{lcccc}
\hline$\tau_{1,6}^{\prime 2}$ & $\tau_{2,7}^{\prime 2}$ & $\tau_{3,8}^{\prime 2}$ & $\tau_{4,9}^{\prime 2}$ & $\tau_{5,10}^{\prime 2}$ \\
\hline$-0.0003 \mp 0.0002 i$ & $-0.0077 \pm 0.0073 i$ & $+0.0658 \pm 0.0023 i$ & $-0.3467 p 0.0447 i$ & $-2.3819 \mp 1.0753 i$ \\
\hline
\end{tabular}

TABle 7: Matrix $\psi^{\prime}$.

\begin{tabular}{ccccc}
\hline$\psi_{1,6}^{\prime}$ & $\psi_{2,7}^{\prime}$ & $\psi_{3,8}^{\prime}$ & $\psi_{4,9}^{\prime}$ & $\psi_{5,10}^{\prime}$ \\
\hline$+0.0210 \mp 0.0210 i$ & $+0.0353 \pm 0.0353 i$ & $-0.0410 \mp 0.0410 i$ & $+0.0357 \pm 0.0357 i$ & $+0.0203 \pm 0.0203 i$ \\
$+0.0574 \mp 0.0574 i$ & $+0.0657 \pm 0.0657 i$ & $-0.0283 \mp 0.0283 i$ & $-0.0201 \mp 0.0201 i$ & $-0.0329 \mp 0.0329 i$ \\
$+0.0918 \mp 0.0918 i$ & $+0.0470 \pm 0.0470 i$ & $+0.0335 \pm 0.0335 i$ & $-0.0221 \mp 0.0221 i$ & $+0.0316 \pm 0.0316 i$ \\
$+0.1183 \mp 0.1183 i$ & $-0.0115 \mp 0.0115 i$ & $+0.0346 \pm 0.0346 i$ & $+0.0374 \pm 0.0374 i$ & $-0.0199 \mp 0.0199 i$ \\
$+0.1353 \mp 0.1353 i$ & $-0.0754 \mp 0.0754 i$ & $-0.0440 \mp 0.0440 i$ & $-0.0215 \mp 0.0215 i$ & $+0.0079 \pm 0.0079 i$ \\
\hline
\end{tabular}

TABLE 8: Identified normalized eigenvectors $\phi_{k}$.

\begin{tabular}{lcccc}
\hline$\phi_{1}$ & $\phi_{2}$ & $\phi_{3}$ & $\phi_{4}$ & $\phi_{5}$ \\
\hline+0.0166 & +0.0499 & +0.0793 & +0.0869 & +0.0582 \\
+0.0454 & +0.0930 & +0.0548 & -0.0489 & -0.0943 \\
+0.0726 & +0.0665 & -0.0649 & -0.0537 & +0.0907 \\
+0.0935 & -0.0163 & -0.0669 & +0.0908 & -0.0570 \\
+0.1069 & -0.1068 & +0.0852 & -0.0522 & +0.0228 \\
\hline
\end{tabular}

dof where the data are missed. Also, only reduced form of the physical matrices can be derived from (25). The results are the $m \times m$ reduced form obtained by removing from each of (37) and (38) the second column and the second row. The reduced stiffness $\widehat{\mathbf{K}}$ matrix can be also derived by using (26), valid in the case of general damping. It results in the following:

$$
\widehat{\mathbf{K}}=10^{5}\left[\begin{array}{cccc}
-2.3115 & -0.4295 & 0.0860 & -0.0140 \\
-0.4295 & -1.8435 & 1.1550 & -0.2530 \\
+0.0860 & 1.1550 & -1.4400 & 0.6050 \\
-0.0140 & -0.2530 & 0.6050 & -0.3970
\end{array}\right] .
$$

The comparison with the matrix (38) shows that the reduced stiffness matrix is slightly different from the exact one with differences among the correspondent diagonal terms less than $1 \%$ and those among the off-diagonal terms less than $3 \%$. This means that the reduced stiffness matrix (39) can be considered a good approximation of the exact one.

It is worthy to compare the results of the identification process without and with the optimization procedure described in the paragraph 4 , when the time history accelerations are polluted with a Gaussian, zero-mean, white noise $(10 \%)$. The procedure has been adopted to improve the estimates of the modal parameters (frequencies, dampings, and eigenvectors). In the procedure to minimize the objective function (31) a Levenberg-Marquardt algorithm is used.

Table 9 shows the frequencies and damping coefficients when evaluated using the optimization procedure ( $f^{\mathrm{opt}} \mathrm{e}$ $\left.\zeta^{\text {opt }}\right)$ and without it $(f$ e $\zeta$ ). It is evident that the identification is satisfactory also without the optimization procedure which, however, reduces the differences with respect to reference values. The efficacy of the optimization procedure is also evidenced in the results reported in Tables 10 and 11 in terms of the $\boldsymbol{\theta}_{k}$ and $\boldsymbol{\phi}_{k}$ eigenvectors.

5.2. Experimental Application. The proposed methodology has also been used to identify the modal and physical parameters of the structure shown in Figure 3. The test structure represents a 1:5 scaled adjacent structures model used in a shaking table experimentation on both coupled and uncoupled configurations, Cimellaro et al. [23]. In this paper the attention is focused to the four dofs structure in the uncoupled configuration.

The structure has plan dimension of $60 \times 60 \mathrm{~cm}$ and interstory height of $60 \mathrm{~cm}$, so that the structure is $240 \mathrm{~cm}$ high. The vertical elements have been realized with commercial steel profiles L $40 \times 40 \times 4 \mathrm{~mm}$ and all the connections 


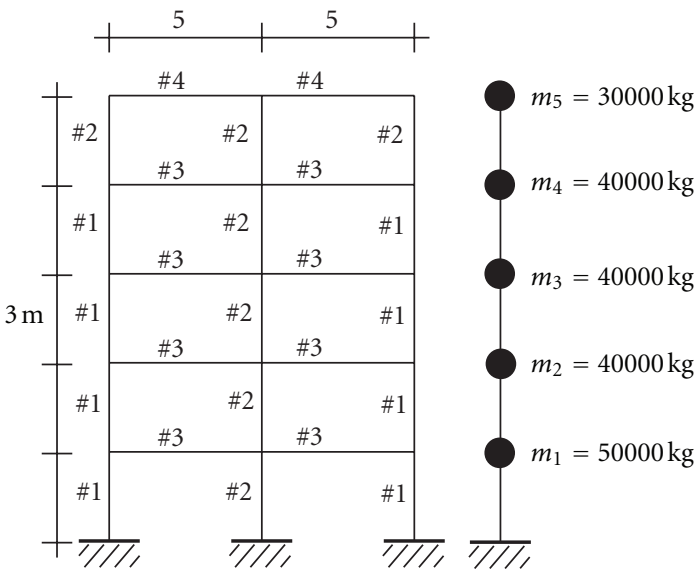

\begin{tabular}{c|c} 
Cross section & Flexural stiffness EJ \\
\hline$\# 1$ & $1.13 \cdot 10^{8} \mathrm{~N} \mathrm{~m}^{2}$ \\
\hline$\# 2$ & $8.45 \cdot 10^{7} \mathrm{~N} \mathrm{~m}^{2}$ \\
\hline$\# 3$ & $4.53 \cdot 10^{7} \mathrm{~N} \mathrm{~m}^{2}$ \\
\hline$\# 4$ & $3.18 \cdot 10^{7} \mathrm{~N} \mathrm{~m}^{2}$
\end{tabular}

Figure 1: Five dofs frame.

TABLE 9: Identified frequencies (Hz) and dampings (\%) without and with optimization.

\begin{tabular}{lccccc}
\hline Modes & 1 & 2 & 3 & 4 & 5 \\
\hline$f$ & 1.252 & 4.015 & 7.499 & 11.828 & 16.518 \\
$f^{\text {opt }}$ & 1.252 & 4.014 & 7.499 & 2.828 & 16.517 \\
$\zeta$ & 2.020 & 2.008 & 2.021 & 2.065 & 2.577 \\
$\zeta^{\text {opt }}$ & 2.000 & 2.000 & 2.000 & 2.010 & 2.050 \\
\hline
\end{tabular}

TABLE 10: Identified seismic eigenvectors $\boldsymbol{\theta}_{k}$ with optimization.

\begin{tabular}{llcccc}
\hline Floor & $\boldsymbol{\theta}_{1}^{\text {opt }}$ & $\boldsymbol{\theta}_{2}^{\text {opt }}$ & $\boldsymbol{\theta}_{3}^{\text {opt }}$ & $\boldsymbol{\theta}_{4}^{\text {opt }}$ & $\boldsymbol{\theta}_{5}^{\text {opt }}$ \\
\hline 1 & +0.2077 & +0.2502 & +0.2718 & +0.1996 & +0.0701 \\
3 & +0.9078 & +0.3347 & -0.2249 & -0.1238 & +0.1073 \\
4 & +1.1698 & -0.0834 & -0.2281 & +0.2102 & -0.0677 \\
5 & +1.3372 & -0.5347 & +0.2923 & -0.1216 & +0.0272 \\
\hline
\end{tabular}

TABLE 11: Identified normalized eigenvectors $\phi_{k}$ with optimization.

\begin{tabular}{|c|c|c|c|c|c|}
\hline Floor & $\phi_{1}^{\mathrm{opt}}$ & $\phi_{2}^{\mathrm{opt}}$ & $\phi_{3}^{\mathrm{opt}}$ & $\phi_{4}^{\mathrm{opt}}$ & $\phi_{5}^{\mathrm{opt}}$ \\
\hline 1 & +0.0166 & +0.0499 & +0.0791 & +0.0866 & +0.0589 \\
\hline 3 & +0.0726 & +0.0667 & -0.0655 & -0.0537 & +0.0901 \\
\hline 4 & +0.0935 & -0.0166 & -0.0664 & +0.0912 & -0.0570 \\
\hline 5 & +0.1069 & -0.1066 & +0.0851 & -0.0527 & +0.0231 \\
\hline
\end{tabular}

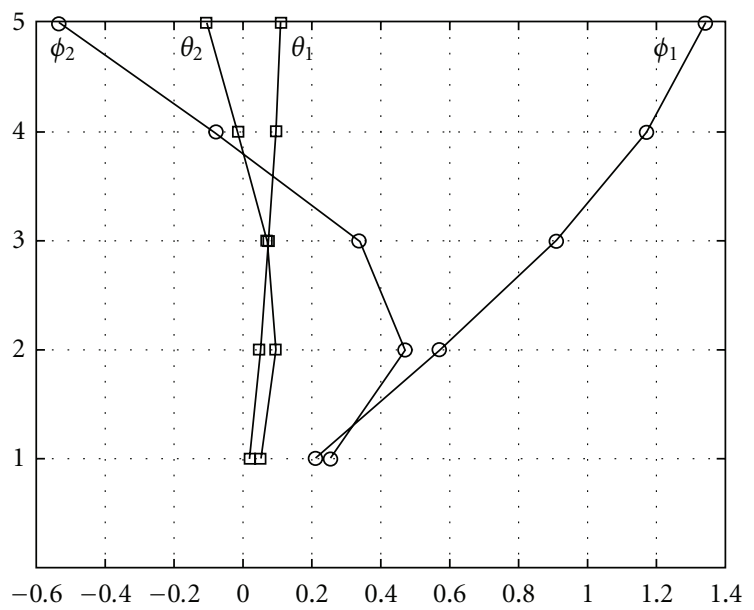

FIGURE 2: First and second identified eigenvectors. 


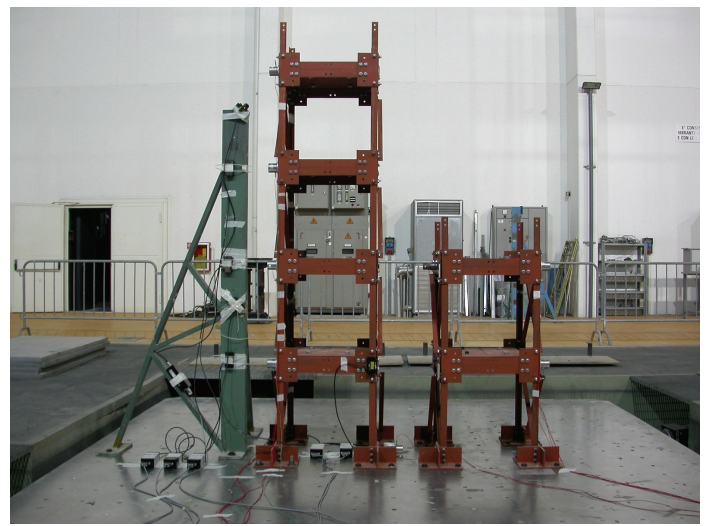

Figure 3: Experimental model.

are bolted. Four steel blocks, of $19 \mathrm{~kg}$ mass each, have been installed at each floor to simulate the floor mass. The total mass of the structure is about $500 \mathrm{~kg}$.

In order to measure the structural response, the test structure has been instrumented with accelerometers at each floor and on the shaking table, and with laser displacement transducers, Figure 3. In the characterization tests several monodirectional excitations (random white noise and natural seismic records), with different intensities have been used. The data acquisition has been made by using an MTS 468D system, with sampling rate of $200 \mathrm{~Hz}$.

The mass matrix is assumed to be diagonal, with $m_{i}=$ $125 \mathrm{~kg}$. In Table 12 the identified frequencies and damping coefficients are reported. The identified damping and stiffness matrices are presented in the following matrices:

$$
\begin{array}{r}
\mathbf{L}=10^{2}\left[\begin{array}{cccc}
7.3899 & -0.9820 & -1.4820 & 0.8828 \\
-0.9820 & 5.5690 & -0.5126 & -0.5928 \\
-1.4820 & -0.5126 & 6.3829 & -1.7643 \\
0.8829 & -0.5928 & -1.7643 & 5.1474
\end{array}\right], \\
\mathbf{K}=10^{6}\left[\begin{array}{cccc}
8.2009 & -4.3274 & 0.3621 & 0.3665 \\
-4.3274 & 8.1550 & -4.7931 & 0.3545 \\
0.3621 & -4.7931 & 8.2536 & -3.8763 \\
0.3665 & 0.3545 & -3.8763 & 3.3340
\end{array}\right] .
\end{array}
$$

Finally, Figure 4 shows, for the El Centro input, the experimental and numerical accelerations at each floor. The figure proves that the identified numerical model reproduces the experimental data very well.

\section{Conclusions}

In this paper a procedure to identify the modal and physical parameters of a linear system subjected to ground motion has been discussed. In particular, the paper has proposed a methodology which is based on a time-domain first-order state representation and allows, first, to identify the modal parameters of the system including the seismic eigenvectors which do not require any normalization. Then, the procedure is able to provide the conditions to normalizing the eigenvectors and, hence, deriving the second-order matrices of the system. The significance of the proposed methodology can be summarized in the following points.

(a) The procedure identifies the seismic eigenvectors of a classically damped system instead of the normal modes (as usually found in the literature) which, in contrast with the normal modes, do not require any normalization and, thus, they can be determined whatever the number of sensors is and with no information and restrictions about the physical model of the system. This means that the seismic eigenvectors can represent a useful tool for health monitoring and of significance in updating models where they provide, jointly with the frequencies and dampings, additional information for validating the model.

(b) In the case of a full set of instrumentations, it provides the conditions to normalizing the eigenvectors and, hence, deriving the second-order matrices of the system without any restrictions about the system behaviour. This represents a new finding with respect to the available literature which instead proposes methodologies valid under the assumption of sheartype behaviour.

(c) In the case of incomplete set of measurements, it is still possible to normalize the eigenvectors with the unique assumption of having at least one mass of the measured dofs known and under the satisfaction of a certain condition (22). In this case, it is also possible to obtain reduced forms of the physical matrices.

(d) The assumption of having at least one known mass at the measured degrees of freedom to identify the physical properties of the system can be easily verified if the procedure is applied to buildings where the evaluation of the floor mass is quite simple. However, it is interesting to observe that the identified matrices $\mathbf{M}, \mathbf{K}$, and $\mathbf{C}$ (see (30)) depend linearly on one unknown mass. This could be useful in the case of finite element updating models as well as in damage detection processes because, in this last case, the comparison between the system's state before damage 
TABLE 12: Identified frequencies and damping coefficients.

\begin{tabular}{lcccc}
\hline Modes & 1 & 2 & 3 & 4 \\
\hline$f(\mathrm{~Hz})$ & 8.30 & 25.34 & 43.11 & 55.61 \\
$\zeta(\%)$ & 2.68 & 1.30 & 1.40 & 0.74 \\
\hline
\end{tabular}

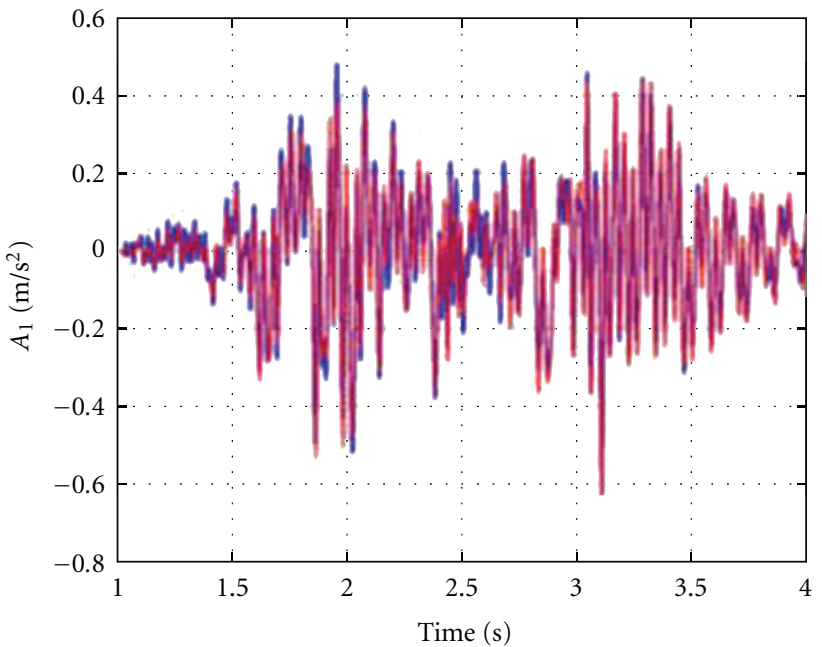

(a)

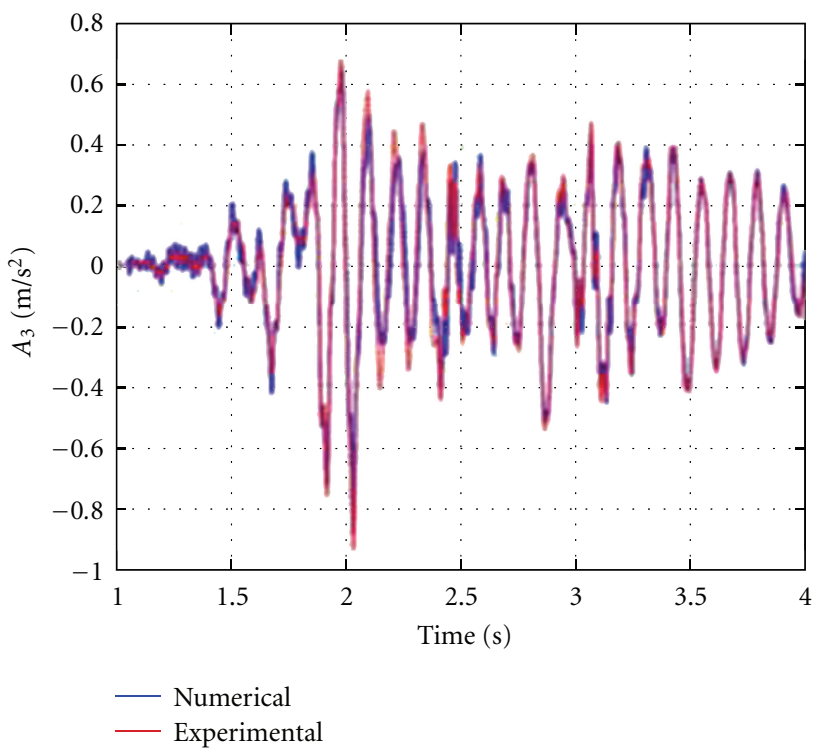

(c)

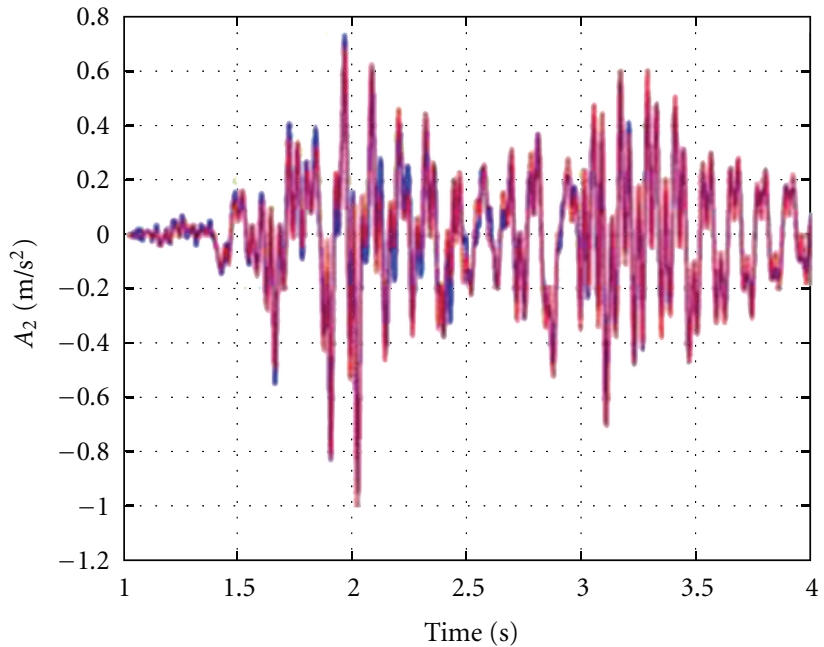

(b)

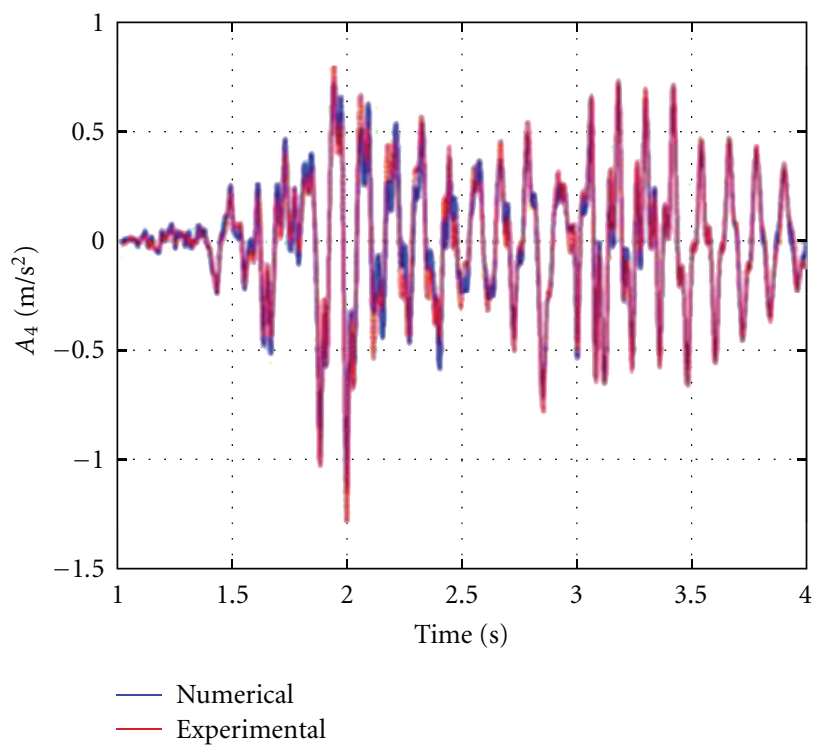

(d)

FIgURE 4: Time histories absolute accelerations: experimental $v s$ numerical.

would be independent on the unknown mass if it is assumed constant.

The procedure has been discussed theoretically and, then, applied to some numerical examples which have shown the efficacy of the procedure. Finally, the procedure has been validated also by an experimental test performed on a fourstory steel frame subjected to earthquake excitations. The authors are actually analyzing the extension of the proposed procedure to nonclassically damped systems.

\section{References}

[1] M. De Angelis, H. Luş, R. Betti, and R. W. Longman, "Extracting physical parameters of mechanical models from identified 
state-space representations," Journal of Applied Mechanics, vol. 69, no. 5, pp. 617-625, 2002.

[2] H. Luş, M. De Angelis, and R. Betti, "A new approach for reduced order modeling of mechanical systems using vibration measurements," Journal of Applied Mechanics, vol. 70, no. 5, pp. 715-723, 2003.

[3] J. M. W. Brownjohn, "Ambient vibration studies for system identification of tall buildings," Earthquake Engineering and Structural Dynamics, vol. 32, no. 1, pp. 71-95, 2003.

[4] H. Imai, C.-B. Yun, O. Maruyama, and M. Shinozuka, "Fundamentals of system identification in structural dynamics," Probabilistic Engineering Mechanics, vol. 4, no. 4, pp. 162-173, 1989.

[5] Ghanem and Shinozuka, "Structural-system identification. I: theory," Journal of Engineering Mechanics, vol. 121, no. 2, pp. 255-264, 1995.

[6] M. Shinozuka and R. Ghanem, "Structural system identification. II: experimental verification," Journal of Engineering Mechanics, vol. 121, no. 2, pp. 265-273, 1995.

[7] J. L. Beck and P. C. Jennings, "Structural identification using linear models and earthquake records," Engineering and Structural Dynamics, vol. 8, no. 2, pp. 145-160, 1980.

[8] G. H. McVerry, "Structural identification in the frequency domain from earthquake records," Earthquake Engineering and Structural Dynamics, vol. 8, no. 2, pp. 161-189, 1980.

[9] U. Füllekrug and J. M. Sinapius, "Identification of modal parameters, generalized and effective masses during basedriven tests," Aerospace Science and Technology, vol. 2, no. 7, pp. 469-480, 1998.

[10] C. H. Loh, C. Y. Lin, and C. C. Huang, "Time domain identification of frames under earthquake loadings," Journal of Engineering Mechanics, vol. 126, no. 7, pp. 693-703, 2000.

[11] S. Y. Chen, M. S. Ju, and Y. G. Tsuei, "Extraction of normal modes for highly coupled incomplete systems with general damping," Mechanical Systems and Signal Processing, vol. 10, no. 1, pp. 93-106, 1996.

[12] P. Yuan, Z. Wu, and X. Ma, "Estimated mass and stiffness matrices of shear building from modal test data," Earthquake Engineering and Structural Dynamics, vol. 27, no. 5, pp. 415421, 1998.

[13] I. Takewaki and M. Nakamura, "Stiffness-damping simultaneous identification using limited earthquake records," Earthquake Engineering and Structural Dynamics, vol. 29, no. 8, pp. 1219-1238, 2000.

[14] K. F. Alvin and K. C. Park, "Second-order structural identification procedure via state-space-based system identification," AIAA journal, vol. 32, no. 2, pp. 397-406, 1994.

[15] K. F. Alvin, L. D. Peterson, and K. C. Park, "Method for determining minimum-order mass and stiffness matrices from modal test data," AIAA journal, vol. 33, no. 1, pp. 128-135, 1995.

[16] H. Luş, R. Betti, and R. W. Longman, "Identification of linear structural systems using earthquake-induced vibration data," Earthquake Engineering and Structural Dynamics, vol. 28, no. 11, pp. 1449-1467, 1999.

[17] G. Fraraccio, A. Brugger, and R. Betti, "Identification and damage detection in structures subjected to base excitation," Experimental Mechanics, vol. 48, no. 4, pp. 521-528, 2008.

[18] H. Luş, Control theory based system identification, Ph.D. thesis, Columbia University, New York, NY, USA, 2001.

[19] D. Capecchi, M. De Angelis, and V. Sepe, "Modal model identification with unknown nonstationary base motion," Meccanica, vol. 39, no. 1, pp. 31-45, 2004.
[20] V. Sepe, D. Capecchi, and M. De Angelis, "Modal model identification of structures under unmeasured seismic excitations," Earthquake Engineering and Structural Dynamics, vol. 34, no. 7, pp. 807-824, 2005.

[21] J. N. Juang, Applied System Identification, Prentice Hall, Upper Saddle River, NJ, USA, 1994.

[22] M. De Angelis, V. Sepe, and F. Vestroni, "Identificazione dei parametri modali di una struttura eccitata alla base," Ingegneria Sismica, vol. 3, pp. 42-50, 2001.

[23] G. P. Cimellaro, M. De Angelis, E. Renzi, and V. Ciampi, "Theory and experimentation on passive control of adjacent structures," in Proceedings of the 13rd World Conference Earthquake Engineering, Vancouver, Canada, August 2004. 

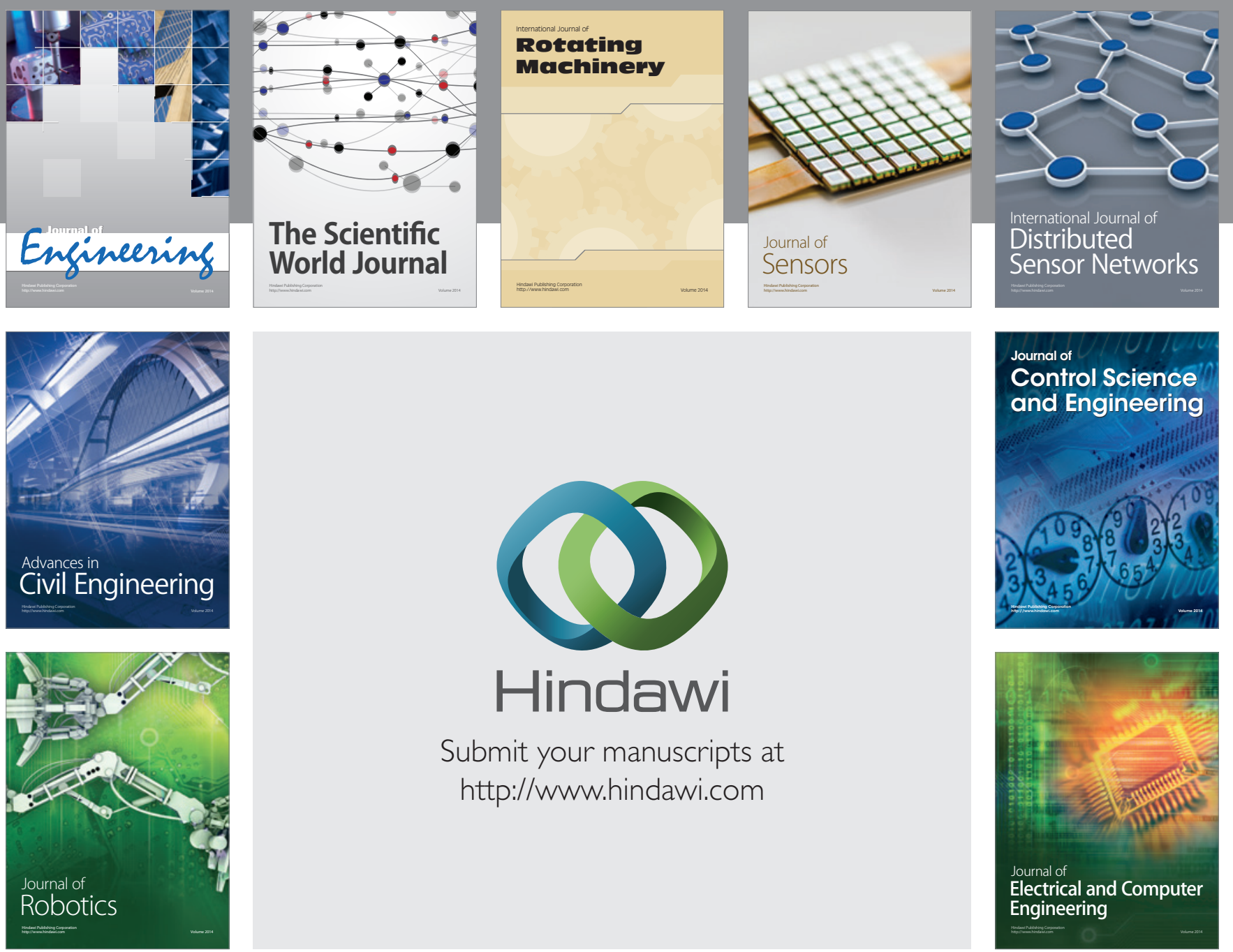

Submit your manuscripts at

http://www.hindawi.com
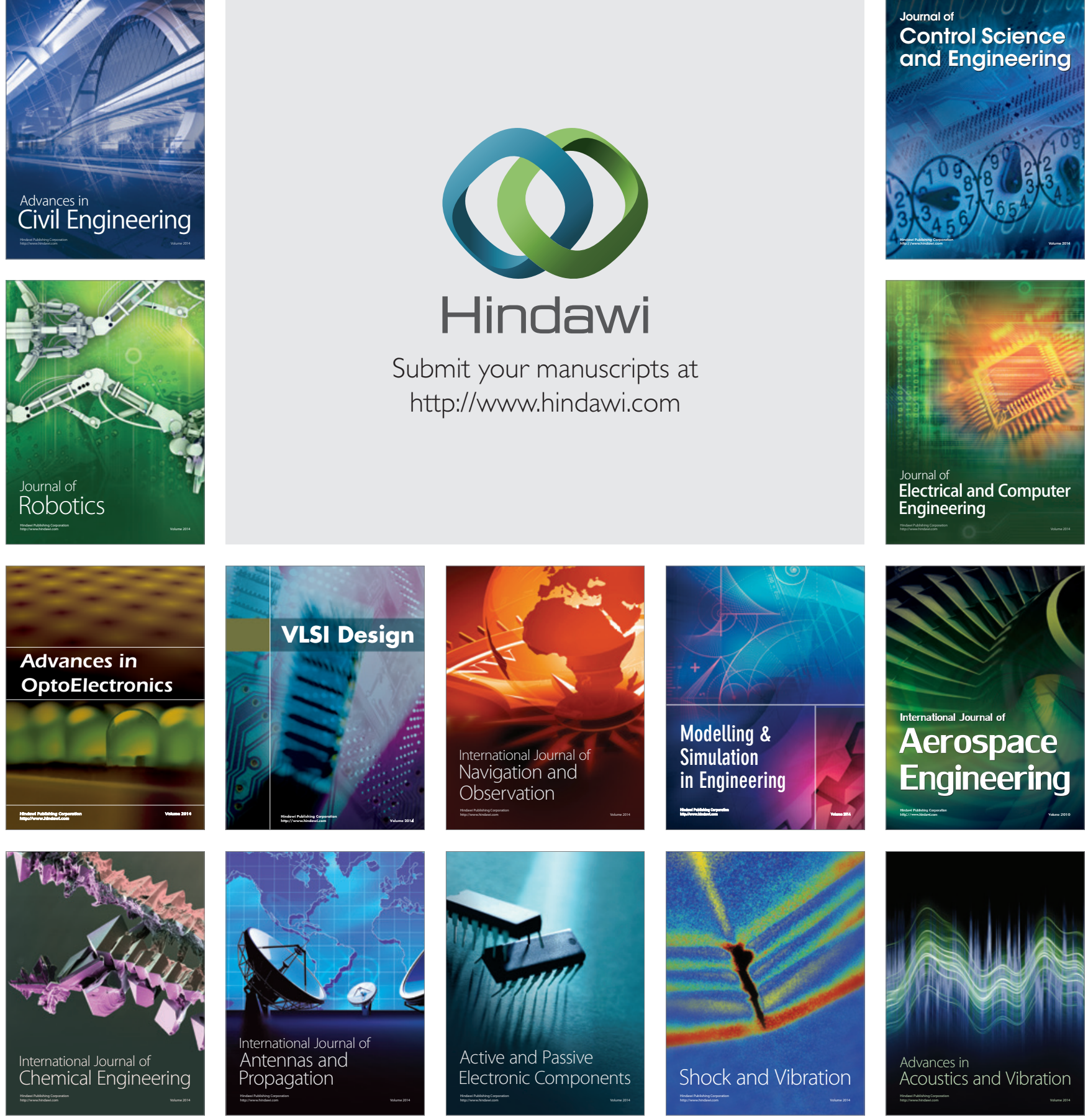